\title{
Development of an Alendronate Controlled Delivery System for Bone Repair Applications
}

\author{
Andreea-Gabriela Deca ${ }^{1}$, Ionela Belu ${ }^{2}$, Octavian Croitoru ${ }^{3}$, Johny Neamţu ${ }^{3}$ \\ ${ }^{1}$ University of Medicine and Pharmacy of Craiova, Faculty of Pharmacy, Romania, Doctoral School. \\ gabriela_deca@yahoo.com \\ ${ }^{2}$ University of Medicine and Pharmacy of Craiova, Faculty of Pharmacy, Romania, Department II of Pharmacy. \\ ${ }^{3}$ University of Medicine and Pharmacy of Craiova, Faculty of Pharmacy, Romania, Department I of Pharmacy.
}

\section{Extended Abstract}

A great interest has been shown towards designing bone targeted medical delivery systems for the treatment of several bone disorders. This approach ensures the release of the drug to the site of the disease [1]. Moreover, it allows delivery of drugs that have low bioavailability when administrated by conventional routes. This leads to enhanced patient adherence and an improved clinical outcome of bone related diseases [2].

Given that hydroxyapatite is a major component of the bone matrix, it represents a target for specific delivery systems [3]. Due to their structure which consists in two phosphonates groups linked to a carbon atom, bisphosphonates show a high affinity for hydroxyapatite. Furthermore they increase both osteoblast proliferation and osteoclast apoptosis leading to bone remodeling [4]. Alendronate is a bisphosphonate used in the treatment and prevention of osteoporosis, Paget's disease, primary hyperparathyroidism, bone metastasis, multiple myeloma [5].

Microparticle-mediated drug delivery to the bone is a promising approach which ensures a high local alendronate concentration and a controlled release of the drug [4, 6]. A recent study conducted by Stadelmann et al. [7] shows an increase in bone density when zoledronate was locally delivered.

Poly lactic-co-glycolic acid (PLGA) is a copolymer approved to be safe in pharmaceutical applications by the FDA due to its biocompatibility and biodegradability. A number of PLGA compounds with different copolymer ratio are used to design microparticles with various properties [8].

The aim of the study is to develop and optimize a PLGA-alendronate microparticles based delivery device, designed to target the bone tissue. This carrier system has the advantages of a high biocompatibility and a controlled release of the incorporated drug. Furthermore, it has the benefit of being a biodegradable system $[8,9]$.

In a first step, the microencapsulation process is to be optimized. There are a number of techniques for PLGA microparticles preparation such as supercritical fluid extraction, extrusion and spray drying [6]. However, the method optimized for this study is the solvent evaporation method. Using this method the microparticles are prepared via a water/oil/water (w/o/w) double emulsion. Initially we prepared two solutions: the alendronate aqueous solution and the PLGA organic solution. The solutions were used to form the primary emulsion which was poured into a polyvinyl alcohol aqueous solution resulting into the w/o/w double emulsion. In order to evaporate the solvent and form the microparticles, the double emulsion was stirred at room temperature for 4 hours. The microparticles obtained following this method remain to be furthermore characterized in order to evaluate the size distribution, entrapment efficiency, morphology and drug release profile. Also, by altering the manufacturing conditions, copolymer ratio and degradation rate, the microparticles drug loading can be adjusted [1].

In a second step, the optimized alendronate loaded microparticles are to be used to develop an implant with high affinity for bone tissue. In conclusion, this system could improve the local delivery of alendronate and have a high potential in bone repair applications.

\section{References}

[1] E. Cenni, D. Granchi, S. Avnet, et al., "Biocompatibility of poly(D,L-lactide-co-glycolide) nanoparticles conjugated with alendronate,” Biomaterials, vol. 29, pp. 1400-141, 2008. 
[2] S. Thamake, S. Raut, Z. Gryczynski, et al., "Alendronate coated poly-lactic-co-glycolic acid (PLGA) nanoparticles for active targeting of metastatic breast cancer," Biomaterials, vol. 33, pp. 7164-7173, 2012.

[3] L. Miguel, M. Noiray, G. Surpateanu, et al., "Poly (-benzyl-l-glutamate)-PEG-alendronate multivalent nanoparticles for bone targeting," Int. J. Pharm., vol. 460, pp. 73-82, 2014.

[4] A. Billon-Chabaud, A. Gouyette, C. Merle, et al., "Development of bisphosphonates controlled delivery systems for bone implantation: influence of the formulation and process used on in vitro release," J. Mater. Sci.: Mater. Med., vol. 21, pp. 1599-1604, 2010.

[5] J. Ezzati, N. Dolatabadi, H. Hamishehkarc, et al., "Formulation, characterization and cytotoxicity studies of alendronatesodium-loaded solid lipid nanoparticles," Colloid Surface B., vol. 117, pp. 21-28, 2014.

[6] K. Derakhshandeh, M. Nikmohammadi, A. Hosseinalizadeh, et al., "Factorial effect of process parameters on pharmaceutical characteristics of biodegradable PLGA microparticles," Int. J. Drug Delivery, vol. 3, pp. 324-334, 2011.

[7] V. Stadelmann, O. Gauthier, A. Terrier, et al., "Implants delivering bisphosphonate locally increase periprosthetic bone density in an osteoporetic sheep model: A pilot study," Eur. Cells Mater., vol. 16, pp. 10-6, 2008.

[8] K. Zhang, X. Tang, J. Zhang, et al., "PEG-PLGA copolymers: Their structure and structure-influenced drug delivery applications," J. Control Release, vol. 183, pp. 77-86, 2014.

[9] X. Shi, Y. Wang, R. Varshney, et al., "Microsphere-based drug releasing scaffolds for inducing osteogenesis of human mesenchymal stem cells in vitro," Eur. J. of Pharm Sci., vol. 39, pp. 59-67, 2010. 